\title{
Introduction to chiral symmetry in QCD
}

\author{
H. Sazdjian ${ }^{1, a}$ \\ ${ }^{1}$ Institut de Physique Nucléaire, CNRS-IN2P3, Université Paris-Sud, Université Paris-Saclay, \\ 91406 Orsay Cedex, France
}

\begin{abstract}
The main aspects of chiral symmetry in QCD are presented. The necessity of its spontaneous breakdown is explained. Some low-energy theorems are reviewed. The role of chiral effective Lagrangians in the formulation and realization of chiral perturbation theory is emphasized. The consequences of the presence of anomalies are sketched.
\end{abstract}

\section{Introduction}

Symmetries play an important role in quantum field theory. (For general surveys, one may consult, e.g., Refs. [1-12].) They introduce limitations in the choice of possible interactions for a given physical problem or phenomenon and often they completely fix the structure of the Lagrangian of the theory. One distinguishes two types of symmetry, local ones, where the parameters of the transformations are spacetime dependent, and global ones, where the latter are spacetime independent. Local symmetries lead in general to the introduction of gauge theories, while global symmetries classify particles according to quantum numbers or predict the existence of massless particles.

$\mathrm{QCD}$, the theory of strong interaction, is a gauge theory with the local symmetry group $S U\left(N_{c}\right)$, acting in the internal space of color degrees of freedom. In the real world, $N_{c}=3$, but leaving $N_{c}$ as a free parameter allows one to study the properties of the theory in more generality. The fundamental fields are the quarks (matter fields) and the gluons (gauge fields).

Quark fields, with a common mass parameter $m$, belong to the defining fundamental representation of the color group, which is $N_{c}$-dimensional, antiquark fields to the complex conjugate representation of the latter $\left(N_{c}\right.$-dimensional), while the gluon fields, which are massless, belong to the adjoint representation $\left(\left(N_{c}^{2}-1\right)\right.$-dimensional).

\section{Flavor symmetry}

QCD is a confining theory, in the sense that quarks and gluons are not observed in nature as individual particles. It is admitted that only gauge invariant objects should be observable. Hadrons are gauge invariant (color singlet) bound states of quarks and gluons and are observed in nature as free particles.

From the spectroscopy of hadrons one deduces that there are several types of quark, currently six, with different masses, having the same properties with respect to the gluon fields. One distinguishes them with a new quantum number, called flavor. One thus has the quarks $u, d, s, c, b, t$.

\footnotetext{
ae-mail: sazdjian@ipno.in2p3.fr
} 
In the present study, we are mainly interested in the flavor sector of quarks. To simplify notation, we omit, in general, the color indices from the quark and gluon fields. The gluons, being flavor singlets, do not have flavor indices. In gauge invariant quantities, an implicit summation on color indices is understood. Like the number of quark colors, $N_{c}$, the number of quark flavors, designated by $N_{f}$, will be considered, for the moment, as a free parameter.

Let us consider the idealized situation where all $N_{f}$ quarks have the same mass $m$. We assign the quark and antiquark fields to the $N_{f}$-dimensional representation and to its complex conjugate one, respectively, with respect to the special unitary group $S U\left(N_{f}\right)$ acting in the internal space of flavors. The quark part of the Lagrangian density becomes

$$
\mathcal{L}_{q}=\bar{\psi}_{a}\left(i \gamma^{\mu}\left(\partial_{\mu}+i g A_{\mu}\right)-m\right) \psi^{a}
$$

(Summation of repeated indices is henceforth assumed: $a$ runs from 1 to $N_{f}$.) It is invariant under the continuous global transformations of the group, with spacetime independent parameters. Designating the latter by $\alpha^{A}\left(A=1, \ldots, N_{f}^{2}-1\right)$, one has in infinitesimal form

$$
\delta \psi^{a}=-i \delta \alpha^{A}\left(T^{A}\right)_{b}^{a} \psi^{b}, \quad \delta \bar{\psi}_{a}=i \delta \alpha^{A} \bar{\psi}_{b}\left(T^{A}\right)_{a}^{b}, \quad \delta A_{\mu}=0
$$

where $T^{A}$ are $N_{f} \times N_{f}$ hermitian traceless matrices; they are the representatives of the generators of the group and hence satisfy the $S U\left(N_{f}\right)$ algebra

$$
\left[T^{A}, T^{B}\right]=i f_{A B C} T^{C}, \quad A, B, C=1, \ldots, N_{f}^{2}-1 .
$$

The $f$ s are called the structure constants of the algebra; they are real and completely antisymmetric in their indices.

Using Noether's theorem, one finds $\left(N_{f}^{2}-1\right)$ conserved currents

$$
j_{\mu}^{A}(x)=-i \frac{\partial \mathcal{L}_{q}}{\partial\left(\partial^{\mu} \psi^{a}\right)}\left(T^{A}\right)^{a}{ }_{b} \psi^{b}=\bar{\psi}_{a} \gamma_{\mu}\left(T^{A}\right)^{a}{ }_{b} \psi^{b}, \quad \partial^{\mu} j_{\mu}^{A}=0
$$

The generators of the group transformations (also called charges) are obtained from $j_{0}^{A}$ by space integration:

$$
Q^{A}=\int d^{3} x j_{0}^{A}(x)
$$

Because of current conservation, the generators are independent of time (the fields are assumed to vanish at infinity) and therefore they commute with the Hamiltonian of the system:

$$
\left[H, Q^{A}\right]=0 .
$$

They satisfy, as operators, the $S U\left(N_{f}\right)$ algebra

$$
\left[Q^{A}, Q^{B}\right]=i f_{A B C} Q^{C}, \quad A, B, C=1, \ldots, N_{f}^{2}-1 .
$$

The system under consideration is thus characterized by the existence of $N_{f}$ quark fields with equal free masses $m$. Since the interaction term itself is invariant under the group transformations, the latter property (equality of masses) is preserved after renormalization.

One might also transcribe the transformation properties of the fields into similar properties of states. Ignoring for the moment the confinement problem and introducing $N_{f}$ one-particle quark 
states $|\mathbf{p}, a\rangle$ (spin labels omitted), created from the vacuum state by the fields $\psi_{a}^{+}$, and assuming the vacuum state is invariant under the transformations of the group, one obtains

$$
Q^{A}\left|\mathbf{p}, a>=\left(T^{A}\right)_{a}^{b}\right| \mathbf{p}, b>.
$$

Equation (6) implies that the various one-particle states of the fundamental representation multiplet have equal masses $m$.

This mode of realization of the symmetry is called the Wigner-Weyl mode.

Other types of relationship can be found between observables involving the multiplet particles, such as form factors or scattering amplitudes.

\section{Approximate flavor symmetry with hadrons}

Consider now the situation where the quarks have different masses, which ultimately corresponds to the real world. The Lagrangian density (1) is replaced by

$$
\mathcal{L}_{q}=\sum_{a=1}^{N_{f}} \bar{\psi}_{a}\left(i \gamma^{\mu}\left(\partial_{\mu}+i g A_{\mu}\right)-m_{a}\right) \psi^{a} .
$$

The mass term of the Lagrangian density is no longer invariant under the transformations of the group $S U\left(N_{f}\right)$ and therefore the currents obtained previously from Noether's theorem are not conserved. One finds

$$
\partial^{\mu} j_{\mu}^{A}=-i \sum_{a, b=1}^{N_{f}}\left(m_{a}-m_{b}\right) \bar{\psi}_{a}\left(T^{A}\right)^{a}{ }_{b} \psi^{b} \neq 0 .
$$

The nonconservation of the currents is thus due to the mass differences within the representation multiplet.

If, however, the mass differences are much smaller than the interaction mass scale, which for QCD is of the order of $\Lambda_{Q C D} \sim 300 \mathrm{MeV}$, or of a multiple of it, one is entitled to treat the effects of the mass differences as perturbations with respect to the symmetric limit where all the masses are equal. One might write the Lagrangian density in the form

$$
\mathcal{L}=\mathcal{L}_{0}+\Delta \mathcal{L}
$$

where $\mathcal{L}_{0}$ is the Lagrangian density with equal masses $m$ and $\Delta \mathcal{L}$ corresponds to mass difference terms.

The above procedure does not destabilize the results of the symmetric theory after renormalization. The reason is that mass operators are soft operators, because their quantized dimension is two for scalar fields and three for fermion fields, smaller than the dimension four of the Lagrangian density and in particular of its interaction part. This has the consequence that mass terms introduce mild effects through renormalization and at the end the latter remain perturbative. In particular, they do not affect the currents with anomalous dimensions [13].

The situation would be different had we introduced the symmetry breaking through the coupling constants of the interaction terms, by assigning a different coupling constant to each flavor type quark. Those have dimension four and their effect on renormalization is hard. At the end, one generally does not find any trace of approximate symmetry, even if at the beginning the coupling constants had been only slightly modified. 
Since hadrons are bound states of quarks and gluons, $S U\left(N_{f}\right)$ flavor symmetry, and more generally its approximate realization, should be reflected in their properties. In the exact symmetry case, hadrons should be classified in $S U\left(N_{f}\right)$ multiplets with degenerate masses.

Considering the real world with six flavor quarks, we observe that they are divided in two groups: the light quarks $u, d, s$ and the heavy quarks $c, b, t$. The mass differences between the two categories being large $(>1 \mathrm{GeV})$, an approximate symmetry can be expected only within the space of the three light quarks. Therefore, flavor symmetry would be concerned either with $S U(2)$ (isospin symmetry), involving the quarks $u$ and $d$, or with $S U(3)$, involving the quarks $u, d, s$.

Concerning isospin symmetry, one notes the approximate equalities of the masses of the proton and the neutron, of the charged and neutral pions, of the kaons and of many other groups of particles. The nucleons and the kaons could be placed in doublet (fundamental) representations of $S U(2)$ (isospin 1/2), the pions in the triplet (adjoint) representation (isospin 1), the $\Delta \mathrm{s}$ in the quadruplet representation (isospin $3 / 2$ ), etc.

Since the mass differences within each multiplet are very small, of the order of a few $\mathrm{MeV}$, one deduces that the difference between the masses of the quarks $u$ and $d$ is also of the same order:

$$
\left|m_{d}-m_{u}\right| \sim \text { a few MeV. }
$$

(Precise calculations should also include the contributions of the electromagnetic interaction, which also are of the same order.)

For the realization of $S U(3)$ symmetry, the quarks $u, d, s$ are assigned to the defining representation 3, while their antiparticles to the representation $\overline{3}$.

Since mesons are made from one quark and one antiquark fields, they would be classified through the product representation

$$
3 \otimes \overline{3}=8 \oplus 1
$$

where 8 is the octet representation and 1 the singlet one. On phenomenological grounds, one notices that for example the vector mesons $\rho, K^{*}, \omega, \phi$ can be grouped in an octet plus a singlet and similarly for the pseudoscalar mesons $\pi, K, \eta, \eta^{\prime}$, although for them the mass differences are much larger than for the vector mesons. (The understanding of the latter phenomenon comes with chiral symmetry).

Baryons, being made from three quark fields, would be classified through the product representation

$$
3 \otimes 3 \otimes 3=10 \oplus 8 \oplus 8 \oplus 1 .
$$

This is the case for example of the baryons $N, \Sigma, \Xi$ and $\Lambda$, which may be classified in an octet, of the baryons $\Delta, \Sigma^{\prime}, \Xi^{\prime}$ and $\Omega$, which may be classified in a decuplet, etc.

The approximate $S U(3)$ symmetry leads to many relations for the mass differences of a given multiplet (Gell-Mann-Okubo formulas), for the coupling constants of particles belonging to multiplets, for form factors, etc.

The mass differences within an $S U(3)$ multiplet being of the order of $100 \mathrm{MeV}$, one deduces that the mass difference between the quark $s$ and the quarks $u$ and $d$ is of the same order of magnitude and much greater than the mass difference between $u$ and $d$ :

$$
\left(m_{s}-\left(m_{u}+m_{d}\right) / 2\right) \sim 100 \mathrm{MeV} \gg\left|\left(m_{d}-m_{u}\right)\right| \sim \text { a few MeV. }
$$

The approximate $S U(3)$ symmetry can also be used for hadrons containing heavy quarks; these, however, should stand as backgrounds for the group analysis. For example one could apply the analysis to mesons made of $\bar{q}_{h} q_{\ell}$, or to baryons made of $q_{h} q_{\ell} q_{\ell}$, where $q_{h}=c, b, t$ and $q_{\ell}=u, d, s$. 


\section{Chiral symmetry}

Fermion fields may undergo other types of unitary transformation than those met with the flavor symmetry. They are generated with the inclusion of the matrix $\gamma_{5}$ in the former transformations and are called axial flavor transformations, since they change the parity properties of fields. In infinitesimal form they are of the type

$$
\delta \psi^{a}=-i \delta \alpha^{A}\left(T^{A}\right)_{b}^{a} \gamma_{5} \psi^{b}, \quad \delta \bar{\psi}_{a}=-i \delta \alpha^{A} \bar{\psi}_{b} \gamma_{5}\left(T^{A}\right)_{a}^{b}, \quad \delta A_{\mu}=0,
$$

where the indices $a, b, A$ refer to the flavor group $S U\left(N_{f}\right)$ representations met before and the $T$ s are hermitian traceless matrices representing the corresponding generators in the fundamental representation.

We consider the quark part of the QCD Lagrangian density with equal mass quarks [Eq. (1)]. Under the above transformations, this Lagrangian density is not invariant, because of the presence of the mass terms:

$$
\delta \mathcal{L}_{q}=2 \operatorname{im} \delta \alpha^{A} \bar{\psi}_{a}\left(T^{A}\right)^{a}{ }_{b} \gamma_{5} \psi^{b} .
$$

Thus, invariance under axial flavor transformations requires vanishing of the quark mass terms. Contrary to the ordinary flavor symmetry transformations, equality of masses is no longer sufficient for ensuring invariance.

In the more general case of unequal masses, the mass terms can be represented in the form of a diagonal matrix $\mathcal{M}$, such that $\mathcal{M}=\operatorname{diag}\left(m_{1}, m_{2}, \ldots, m_{N_{f}}\right)$. In that case the variation of the Lagrangian density is

$$
\delta \mathcal{L}_{q}=i \delta \alpha^{A} \bar{\psi}_{a}\left\{\mathcal{M}, T^{A}\right\}_{b}^{a} \gamma_{5} \psi^{b},
$$

where $\{$,$\} is the anticommutator.$

We now consider the case of massless quarks. The Lagrangian density is invariant under both the flavor and axial flavor transformations. The conserved currents are

$$
\begin{aligned}
& j_{\mu}^{A}(x)=\bar{\psi}_{a} \gamma_{\mu}\left(T^{A}\right)^{a}{ }_{b} \psi^{b}, \quad \partial^{\mu} j_{\mu}^{A}=0, \\
& j_{5 \mu}^{A}(x)=\bar{\psi}_{a} \gamma_{\mu} \gamma_{5}\left(T^{A}\right)^{a}{ }_{b} \psi^{b}, \quad \partial^{\mu} j_{5 \mu}^{A}=0 .
\end{aligned}
$$

The corresponding charges are defined from space integration on the current densities:

$$
Q^{A}=\int d^{3} x j_{0}^{A}(x), \quad Q_{5}^{A}=\int d^{3} x j_{50}^{A}(x)
$$

The flavor and axial flavor transformations form the set of chiral transformations. The corresponding charges satisfy the following algebra:

$$
\begin{aligned}
& {\left[Q^{A}, Q^{B}\right]=i f_{A B C} Q^{C}, \quad\left[Q^{A}, Q_{5}^{B}\right]=i f_{A B C} Q_{5}^{C},} \\
& {\left[Q_{5}^{A}, Q_{5}^{B}\right]=i f_{A B C} Q^{C}, \quad A, B, C=1, \ldots, N_{f}^{2}-1 .}
\end{aligned}
$$

Note that the axial charges do not form alone an algebra. The previous algebra can, however, be simplified and become more transparent. Define

$$
Q_{L}^{A}=\frac{1}{2}\left(Q^{A}-Q_{5}^{A}\right), \quad Q_{R}^{A}=\frac{1}{2}\left(Q^{A}+Q_{5}^{A}\right) .
$$

( $L$ for left-handed and $R$ for right-handed.) One obtains

$$
\left[Q_{L}^{A}, Q_{L}^{B}\right]=i f_{A B C} Q_{L}^{C}, \quad\left[Q_{R}^{A}, Q_{R}^{B}\right]=i f_{A B C} Q_{R}^{C}, \quad\left[Q_{L}^{A}, Q_{R}^{B}\right]=0 .
$$


Therefore, the left-handed and right-handed charges are decoupled and operate separately. Each of them generate an $S U\left(N_{f}\right)$ group of transformations. The whole chiral group is then decomposed into the direct product of two $S U\left(N_{f}\right)$ groups, which will be labeled with the subscripts $L$ and $R$, respectively:

$$
\text { Chiral group }=S U\left(N_{f}\right)_{L} \otimes S U\left(N_{f}\right)_{R} .
$$

The ordinary flavor transformations form a subgroup of these, denoted $S U\left(N_{f}\right)_{V}$ :

$$
\text { Flavor group }=S U\left(N_{f}\right)_{V} \text {. }
$$

(The subscript $V$ refers to the vector nature of the corresponding currents.)

\section{Explicit chiral symmetry breaking}

In nature, quarks have masses. Therefore, chiral symmetry cannot be an exact symmetry of the QCD Lagrangian. The quark mass terms introduce an explicit chiral symmetry breaking.

The symmetry breaking could be treated as a perturbation only if the quark masses are much smaller than the QCD mass scale. This eliminates the heavy quarks $c, b, t$ from the domain of investigations. We are left with the sector of light quarks $u, d, s$ and the approximate chiral symmetry $S U(3)_{L} \otimes S U(3)_{R}$.

What would be the signature of this approximate symmetry in nature? The axial charges $Q_{5}^{A}$ are pseudoscalar objects. When acting, in the chiral symmetry limit (massless quarks) and in the WignerWeyl mode, on a massive hadronic state belonging to a multiplet of the flavor group, they would produce new states with the same mass and spin, but with opposite parity:

$$
Q_{5}^{A}\left|M, s, \mathbf{p},+, a>=\left(T^{\prime A}\right)^{b}\right| M, s, \mathbf{p},-, b>.
$$

The + and - labels refer to the intrinsic parity of the states; for definiteness, we have assumed above that the initial state has positive parity. (For chiral singlet representations the matrix $T^{\prime}$ is null.)

Thus, if we adopt the Wigner-Weyl mode of realization of chiral symmetry, we should find parity doublets for most of massive states.

When the light quarks obtain masses, the degeneracy of masses within the parity doublets would be removed, but the masses would still remain close to each other. However, no parity doublets of massive particles with approximately equal masses are found, in general, in the hadronic world. This observation forces us to abandon the Wigner-Weyl mode of realization of chiral symmetry.

The other alternative that remains is the phenomenon of spontaneous symmetry breaking, also called the Nambu-Goldstone mode of realization of chiral symmetry [14-17].

\section{Spontaneous chiral symmetry breaking}

An inherent assumption within the Wigner-Weyl mode is that the ground state of the theory (the vacuum state in QFT) is invariant under the symmetry group of transformations. This means that the generators of the transformations annihilate the vacuum state:

$$
Q^{A} \mid 0>=0 .
$$

(Here, the specific case of vector flavor charges has been considered.)

Since one-particle states are constructed from the action of the fields on the vacuum state, the above property guarantees that one-particle states do transform as elements of irreducible representations of the symmetry group. 
However, the vacuum state (the ground state) may not be invariant under the symmetry group of transformations, even if the Lagrangian is. In that case, it is not a symmetric state and the generators of the symmetry group do not annihilate it:

$$
Q_{5}^{A} \mid 0>\neq 0 .
$$

(Here, the specific case of axial-vector flavor charges has been considered.) It is said that the symmetry is spontaneously broken.

A similar situation is found in the well-known sigma model [18] or in the $O(N)$ model of spin-0 fields [6]. Here, one studies the properties of the potential energy at the classical level. According to the values of the parameters of theory, the potential energy may have a non-symmetric ground state.

The absence of parity doublets for massive hadronic states forces us to explore the possibility of spontaneous breaking of chiral symmetry. The axial charges, when applied on the vacuum state, would now produce new states:

$$
Q_{5}^{A}|0>=| \chi^{A},->, \quad A=1, \ldots, 8 .
$$

These states have the same quantum properties as their generating axial charges, the vacuum state being assumed to have a positive parity and no quantum numbers. In particular, they are pseudoscalar states. Since in the symmetric limit the charges commute with the Hamiltonian, the energy of the states $\mid \chi^{A},->$ is null. This is possible only if there exist massless pseudoscalar particles, which might also generate by superposition other many-particle zero-energy states. This is the content of the Goldstone theorem $[14,15]$. We note, however, that the states $\mid \chi^{A},->$ are not one-particle states, but rather a superposition of many-massless-particle states; their norm is infinite. The fact that these states do not form new degenerate vacua, but rather represent zero-energy limits of many-massless-particle states, hinges on the assumption that the hadronic vacuum state $\mid 0>$ is unique. This is corroborated by the fact that when the quarks obtain small masses, explicitly breaking chiral symmetry, these particles become massive, although with small masses, while the state $\mid 0>$ evolves towards the true vacuum state of the theory.

Spontaneous chiral symmetry breaking is thus manifested by the existence of eight pseudoscalar massless particles (mesons), called Nambu-Goldstone bosons.

Spontaneous breaking concerns, however, only the axial sector of the charges. The ordinary flavor symmetry is still realized with the Wigner-Weyl mode. Therefore the symmetry group $S U(3)_{L} \otimes$ $S U(3)_{R}$ is spontaneously broken down to the flavor group $S U(3)_{V}$ :

$$
S U(3)_{L} \otimes S U(3)_{R} \longrightarrow S U(3)_{V} .
$$

In the real world, where quarks have masses, chiral symmetry will undergo an additional explicit symmetry breaking. Under this effect, the eight Nambu-Goldstone bosons will acquire small masses, as compared to the masses of massive hadrons.

Considering the spectroscopy of mesons, one notices the existence of eight light pseudoscalar mesons, $\pi, K, \eta$. They can be identified, in the chiral limit, with the eight Nambu-Goldstone bosons.

One would expect that the masses-squared of these particles are proportional to the masses of the quarks that make their content, which would explain in turn the mass differences and hierarchies between them. Thus, one should have (cf. Sect. 8.6)

$$
M_{P}^{2}=O(\mathcal{M})
$$

while for the other hadronic massive states, one should have the expansion

$$
M_{h}^{2}=M_{h 0}^{2}+O(\mathcal{M})
$$


$M_{h 0}^{2}$ being the same for all members of a flavor multiplet and different from zero in the chiral limit. ( $M_{h 0}^{2}$ differs from one flavor multiplet to another.) This explains why the lightest pseudoscalar meson masses are more sensitive to the quark masses than those of the massive hadrons.

\section{Properties of Goldstone bosons}

Consider, in the chiral limit, the coupling of the Goldstone bosons to the axial-vector currents:

$$
<0\left|j_{5 \mu}^{A}\right| P^{B}, p>=i \delta_{A B} p_{\mu} F_{P 0} .
$$

The coupling $F_{P 0}$ is different from zero, since otherwise the axial charges, which are constructed from $j_{50}^{A}$, could not create from the vacuum state zero-energy states. Using conservation of the axial vector currents and the operator equation $\partial^{\mu} j_{5 \mu}^{A}=i\left[P_{\mu}, j_{5 \mu}^{A}\right]$, one obtains

$$
<0\left|\partial^{\mu} j_{5 \mu}^{A}\right| P^{B}, p>=0=\delta_{A B} p^{2} F_{P 0}=\delta_{A B} M_{P 0}^{2} F_{P 0},
$$

which implies that $M_{P 0}=0$. This is another verification of the Goldstone theorem.

Consider now the coupling of a massive pseudoscalar state (for example a radial excitation of the Goldstone boson) to the axial-vector current:

$$
<0\left|j_{5 \mu}^{A}\right| P^{\prime B}, p>=i \delta_{A B} p_{\mu} F_{P^{\prime} 0} .
$$

Using conservation of the axial-vector currents one again finds

$$
<0\left|\partial^{\mu} j_{5 \mu}^{A}\right| P^{\prime B}, p>=0=\delta_{A B} p^{2} F_{P^{\prime} 0}=\delta_{A B} M_{P^{\prime} 0}^{2} F_{P^{\prime} 0} .
$$

However, since $M_{P^{\prime} 0} \neq 0$, one deduces that $F_{P^{\prime} 0}=0$. This means that the massive pseudoscalar states decouple from the axial-vector currents.

The above results can be summarized as follows:

Goldstone bosons : $\quad M_{P 0}^{2}=0, \quad F_{P 0} \neq 0$.

Massive pseudoscalar mesons : $\quad M_{P^{\prime} 0}^{2} \neq 0, \quad F_{P^{\prime} 0}=0$.

When quarks obtain masses, the above properties are modified by terms proportional to the quark masses.

Goldstone bosons : $\quad M_{P}^{2}=O(\mathcal{M}), \quad F_{P}=F_{P 0}+O(\mathcal{M})$,

Massive pseudoscalar mesons : $M_{P^{\prime}}^{2}=M_{P^{\prime} 0}^{2}+O(\mathcal{M}), \quad F_{P^{\prime}}=O(\mathcal{M})$,

$M_{P}^{2} \ll M_{P^{\prime}}^{2}, \quad\left|F_{P^{\prime}}\right| \ll\left|F_{P}\right|$.

On experimental grounds, from the leptonic decays of $\pi$ and $K$ mesons, one has [19]

$$
F_{\pi} \simeq 92 \mathrm{MeV}, \quad F_{K} \simeq 110 \mathrm{MeV} .
$$

The quantity $\left(F_{K} / F_{\pi}-1\right) \simeq 0.2$ measures the order of magnitude of flavor $S U(3)$ breaking. 


\section{Low-energy theorems}

The decoupling of the massive pseudoscalar mesons from the axial-vector currents in the chiral limit (massless quarks) allows one to derive low-energy theorems concerning processes where enters at least one Goldstone boson. Most of these relations are obtained with the aid of the Ward-Takahashi identities.

Contrary to to the ordinary flavor symmetry, chiral symmetry does not yield linear relations between matrix elements of multiplets, but rather leads to relations between processes involving absorption and/or emission of Goldstone bosons at low momenta, for example between the process $\alpha \rightarrow \beta$ and the process $\alpha+n_{1} P \rightarrow \beta+n_{2} P$, where $n_{1}$ Goldstone bosons are absorbed and $n_{2}$ Goldstone bosons are emitted $\left(n_{1} \geq 0, n_{2} \geq 0\right)$.

Remark. In QCD, the quark masses appear as free parameters. Therefore, one expects that all hadronic physical quantities - masses, decay couplings, coupling constants, form factors, scattering amplitudes - possess analyticity properties in them, up to the existence of cuts or branching points. These objects appear in general as residues of Green's functions at physical particle poles. Therefore, they define on-mass shell quantities. They should not be considered as functions of the mass-shell variables $p^{2}, p^{\prime 2}$, etc., but only of the quark mass parameters and of the momentum transfers or of the Mandelstam variables $s, t, u$, etc., which, eventually may take unphysical values by analytic continuation. Green's functions, on the other hand, may be functions of the mass-shell variables $p^{2}, p^{\prime 2}$, etc.

\subsection{Goldberger-Treiman relation}

Consider, in the isospin limit, the matrix element of the axial-vector current between proton and neutron states [20]:

$$
<p\left(p^{\prime}\right)\left|j_{5 \mu}^{1+i 2}\right| n(p)>=\bar{u}_{p}\left(p^{\prime}\right)\left[\gamma_{\mu} \gamma_{5} g_{A}\left(q^{2}\right)+q_{\mu} \gamma_{5} h_{A}\left(q^{2}\right)\right] u_{n}(p),
$$

where $q=\left(p-p^{\prime}\right) ; g_{A}$ and $h_{A}$ are the axial-vector form factors of the nucleons.

Taking the divergence of the current, one obtains

$$
<p\left(p^{\prime}\right)\left|\partial^{\mu} j_{5 \mu}^{1+i 2}\right| n(p)>=-i\left(2 M_{N} g_{A}\left(q^{2}\right)+q^{2} h_{A}\left(q^{2}\right)\right) \bar{u}_{p}\left(p^{\prime}\right) \gamma_{5} u_{n}(p) .
$$

The left-hand side has singularities through the contribution of pseudoscalar intermediate states. For simplicity and illustrative purposes, let us assume that the latter can be saturated by a series of narrowwidth particles, composed of the pion $(\pi)$ and of its radial excitations $\left(\pi^{n}, n=1,2, \ldots\right)$. It can be shown that multipion states, because of phase space, do not contribute to the final result. One obtains

$$
<p\left(p^{\prime}\right)\left|\partial^{\mu} j_{5 \mu}^{1+i 2}\right| n(p)>=-2 i\left\{\frac{M_{\pi}^{2} F_{\pi}}{M_{\pi}^{2}-q^{2}} g_{\pi N N}+\sum_{n=1}^{\infty} \frac{M_{\pi^{n}}^{2} F_{\pi^{n}}}{M_{\pi^{n}}^{2}-q^{2}} g_{\pi^{n} N N}\right\} \bar{u}_{p}\left(p^{\prime}\right) \gamma_{5} u_{n}(p),
$$

where $g_{\pi N N}$ and $g_{\pi^{n} N N}$ are the coupling constants of the pseudoscalar mesons with the nucleons. Equation (45) becomes

$$
2 \frac{M_{\pi}^{2} F_{\pi}}{M_{\pi}^{2}-q^{2}} g_{\pi N N}+2 \sum_{n=1}^{\infty} \frac{M_{\pi^{n}}^{2} F_{\pi^{n}}}{M_{\pi^{n}}^{2}-q^{2}} g_{\pi^{n} N N}=2 M_{N} g_{A}\left(q^{2}\right)+q^{2} h_{A}\left(q^{2}\right) .
$$

We take the limit $q^{2}=0$. In the right-hand side, $h_{A}\left(q^{2}\right)$ does not have a pole at this value (no massless pseudoscalars in the real world). This gives

$$
F_{\pi} g_{\pi N N}+\sum_{n=1}^{\infty} F_{\pi^{n}} g_{\pi^{n} N N}=M_{N} g_{A}(0)
$$


Consider now the $S U(2)_{L} \otimes S U(2)_{R}$ chiral limit (massless $u$ and $d$ quarks). According to Eq. (39), all massive pseudoscalar mesons decouple from the axial-vector current. Equation (48) reduces to the relation

$$
g_{A}(0)=\frac{F_{\pi} g_{\pi N N}}{M_{N}}
$$

This is an exact result of QCD in the $S U(2)_{L} \otimes S U(2)_{R}$ chiral limit (a low-energy theorem). Note that it is independent of the order of the two limits that were taken. Had we first considered the chiral limit, then the left-hand side of Eq. (45) would vanish, but at the same time in the right-hand side the form factor $h_{A}\left(q^{2}\right)$ would display a pion pole at the position $q^{2}=0$ and would contribute to the equation.

The experimental values are: $g_{A} \simeq 1.27$ [19], $g_{\pi N N} \simeq 13.15$ [21], $F_{\pi} \simeq 92.2 \mathrm{MeV}$ [19], $M_{N}=$ $\left(M_{p}+M_{n}\right) / 2=938.92 \mathrm{MeV}$. The right-hand side is then $\simeq 1.29$, to be compared with the left-hand side, 1.27. The discrepancy is about $2 \%$, which is typical of the corrections coming from explicit breaking of $S U(2)_{L} \otimes S U(2)_{R}$ symmetry $\left(M_{\pi}^{2} / M_{N}^{2} \simeq 0.02\right)$.

\subsection{Ward-Takahashi identities}

Ward-Takahashi identities (WTI) $[22,23]$ are obtained by considering correlation functions of axialvector currents with local operators $O(x)$ :

$$
\int d x e^{i q \cdot x}<\beta\left|T j_{5 \mu}^{A}(x) O(0)\right| \alpha>
$$

where $\mid \alpha>$ and $\mid \beta>$ are hadronic states.

Taking the divergence of the axial-vector current gives

$$
\begin{aligned}
& -i q^{\mu} \int d x e^{i q \cdot x}<\beta\left|T j_{5 \mu}^{A}(x) O(0)\right| \alpha>=\int d x e^{i q \cdot x}<\beta\left|T \partial^{\mu} j_{5 \mu}^{A}(x) O(0)\right| \alpha> \\
& +\int d x e^{i q \cdot x} \delta\left(x^{0}\right)<\beta\left|\left[j_{50}^{A}(x), O(0)\right]\right| \alpha>.
\end{aligned}
$$

One notices the presence of the equal-time commutator, which should be evaluated in the theory. Because of causality, it should involve a linear combination of $\delta^{3}(\mathbf{x})$ and a finite number of its derivatives.

One then takes the limit of low or zero values of $q$ and proceeds with similar methods as in the Goldberger-Treiman case.

The method can also be generalized by considering correlation functions of the axial-vector currents with a multiple number of local operators.

\subsection{Callan-Treiman relation}

Choose $|\alpha>=| K^{+}>,|\beta>=| 0>, O=j_{v}^{4-i 5}$ and $j_{5 \mu}^{A}=j_{5 \mu}^{3}$ [24]. The equal-time commutator of the WTI yields the axial-vector current $j_{5 v}^{4-i 5}$ plus Schwinger terms that do not contribute. The matrix element involving the divergence of the axial-vector current is again saturated by the pion and its radial excitations. The corresponding residues are proportional to the $K_{\ell 3}$ form factors with respect to the pion and to its radial excitations.

One has the definition

$$
<\pi^{0}\left(p^{\prime}\right)\left|j_{v}^{4-i 5}\right| K^{+}(p)>=\frac{1}{\sqrt{2}}\left[\left(p+p^{\prime}\right)_{v} f_{+}(t)+\left(p-p^{\prime}\right)_{v} f_{-}(t)\right], \quad t=\left(p-p^{\prime}\right)^{2},
$$


with similar definitions for the radial excitations of the pion. Take the limit $q \rightarrow 0$ in the WTI [Eq. (51)], in which case the left-hand side vanishes (no poles at $q=0$ ). Then the $S U(2)_{L} \otimes S U(2)_{R}$ chiral limit is taken (massless $u$ and $d$ quarks). The massive pseudoscalar states decouple and one ends up with the relation

$$
f_{+}\left(M_{K}^{2}\right)+f_{-}\left(M_{K}^{2}\right)=\frac{F_{K}}{F_{\pi}} .
$$

The physical domain of the $K_{\ell 3}$ form factors (corresponding to the decay $K \rightarrow \pi \ell v$ ) being limited by the inequalities $m_{\ell}^{2} \leq t \leq\left(M_{K}-M_{\pi}\right)^{2}$, the form factors appearing in the above relation are evaluated at the unphysical point $t=M_{K}^{2}$. Extrapolations are used from the physical domain to reach that point. The relation is well satisfied on experimental grounds with a few percent of discrepancy.

The Callan-Treiman formula establishes a relation between the form factors of the process $K \rightarrow$ $\pi \ell v$ and the decay coupling of the process $K \rightarrow \ell v$.

\subsection{Pion scattering lengths (Weinberg)}

Choose for $\mid \alpha>$ and $\mid \beta>$ in the WTI [Eq. (51)] target particles like $N, K$ or $\pi$, and for $O$ and $j_{5 \mu}^{A}$ axial vector currents with the pion quantum numbers [25]. Then calculate the divergences of the two currents. The WTI involves now two momenta $q$ and $k$ and two equal time commutators. The momenta squared $q^{2}$ and $k^{2}$ are taken to zero, but $q$ and $k$ are maintained nonzero, of the order of $M_{\pi}$. One ends up with formulas for the S-wave scattering lengths of the processes $\pi+\alpha \rightarrow \pi+\alpha$, where $\alpha$ is the target particle, much heavier than the pion:

$$
a_{0}^{I}=-\frac{M_{\pi}}{8 \pi F_{\pi}^{2}}\left(1+\frac{M_{\pi}}{M_{\alpha}}\right)^{-1}\left[I(I+1)-I_{\alpha}\left(I_{\alpha}+1\right)-2\right],
$$

where $I$ is the total isospin of the state $\mid \pi \alpha>$ and $I_{\alpha}$ the isospin of the target particle. This formula is applied to the scattering processes $\pi+N \rightarrow \pi+N$ and $\pi+K \rightarrow \pi+K[25,26]$.

When the target particle is the pion itself, the analysis should be completed by retaining higherorder terms in the kinematic variables. Crossing symmetry is also used. One then obtains the $\pi-\pi$ scattering amplitude at low energies:

$$
\mathcal{M}_{a c, b d}=\frac{1}{F_{\pi}^{2}}\left\{\delta_{a c} \delta_{b d}\left(s-M_{\pi}^{2}\right)+\delta_{a b} \delta_{c d}\left(t-M_{\pi}^{2}\right)+\delta_{a d} \delta_{b c}\left(u-M_{\pi}^{2}\right)\right\},
$$

where $a, b, c, d$ are the pion isospin indices and $s, t, u$ the Mandelstam variables.

The $\mathrm{S}$-wave scattering lengths are

$$
a_{0}^{0}=\frac{7 M_{\pi}}{32 \pi F_{\pi}^{2}} \simeq 0.16 M_{\pi}^{-1}, \quad a_{0}^{2}=-\frac{2 M_{\pi}}{32 \pi F_{\pi}^{2}} \simeq-0.046 M_{\pi}^{-1} .
$$

The above predictions are well satisfied experimentally within $10-25 \%$ of discrepancy. Direct measurements of the scattering lengths are, however, not possible because of the instability of the pion under weak or electromagnetic interactions. Elaborate extrapolation procedures are used for the extraction of the scattering lengths from high-energy data.

\subsection{Adler-Weisberger relation}

The starting point is the same as for the calculation of the scattering lengths [Sect. 8.4]. One chooses for $\mid \alpha>$ and $\mid \beta>$ in the WTI nucleon states and for $O$ and $j_{5 \mu}^{A}$ axial-vector currents with the pion 
quantum numbers $[27,28]$. The corresponding two momenta $q$ and $k$ are taken to zero. In this limit, there is in addition a nucleon pole that contributes, yielding as a residue the axial-vector form factor of the nucleon at zero momentum transfer. At the end of the operations, one obtains the isospin antisymmetric part of the pion-nucleon scattering amplitude at an unphysical point. The latter is reexpressed by means of a dispersion relation in terms of an integral over physical pion-nucleon cross sections. The final formula is

$$
g_{A}^{2}=1-\frac{2 F_{\pi}^{2}}{\pi} \int_{v_{0}}^{\infty} \frac{d v}{v}\left[\sigma^{\pi^{-} p}(v)-\sigma^{\pi^{+} p}(v)\right], \quad v=q . p .
$$

The right-hand side yields for $g_{A}$ the value 1.24, to be compared with its experimental value 1.27.

\subsection{Gell-Mann-Oakes-Renner formulas}

Choose in the WTI [Eq. (51)] $|\alpha>=| \beta>=\mid 0>$ and for $O$ the divergence of an axial-vector current [29]. One has

$$
\partial^{v} j_{5 v}^{B}=i \bar{\psi}_{a}\left\{\mathcal{M}, T^{B}\right\}_{b}^{a} \gamma_{5} \psi^{b} .
$$

The operators $v^{B}=-i \bar{\psi}_{a}\left(T^{B}\right)^{a}{ }_{b} \gamma_{5} \psi^{b}$ define the pseudoscalar densities. They transform, in the chiral limit, under the action of the axial charges as

$$
\left[Q_{5}^{A}, v^{B}\right]=i d_{A B C} u^{C}+i \frac{1}{3} \delta_{A B} u^{0},
$$

where the $u$ s are the scalar densities

$$
u^{C}=\bar{\psi}_{a}\left(T^{C}\right)_{b}^{a} \psi^{b}, \quad C=1, \ldots, 8, \quad u^{0}=\bar{\psi}_{a} \psi^{a},
$$

and the coefficients $d$ are fully symmetric in their indices; they result from the anticommutators of the matrices $T:\left\{T^{A}, T^{B}\right\}=d_{A B C} T^{C}+\frac{1}{3} \delta_{A B} \mathbf{1}$.

The WTI takes the form

$$
\begin{aligned}
& -i q^{\mu} \int d x e^{i q \cdot x}<0\left|T j_{5 \mu}^{A}(x) \partial^{v} j_{5 v}^{B}\right| 0>=\int d x e^{i q \cdot x}<0\left|T \partial^{\mu} j_{5 \mu}^{A}(x) \partial^{v} j_{5 v}^{B}\right| 0> \\
& +\int d x e^{i q \cdot x} \delta\left(x^{0}\right)<0\left|\left[j_{50}^{A}(x), \partial^{v} j_{5 v}^{B}\right]\right| 0>.
\end{aligned}
$$

Intermediate states are only pseudoscalar mesons. In the limit $q=0$ there are no poles to contribute in the left-hand side and the latter vanishes, yielding

$$
\delta_{A B}\left\{M_{P^{A}}^{2} F_{P^{A}}^{2}+\sum_{n=1}^{\infty} M_{P^{n A}}^{2} F_{P^{n A}}^{2}\right\}=-\operatorname{tr}\left\{T^{A},\left\{\mathcal{M}, T^{B}\right\}\right\}<0\left|\frac{1}{3} u^{0}\right| 0>.
$$

From Eqs. (40) and (41) we deduce that $F_{P^{A n}}^{2}=O\left(\mathcal{M}^{2}\right)$, which is much smaller than $O(\mathcal{M})$ terms. Keeping only $O(\mathcal{M})$ quantities, one obtains:

$$
\delta_{A B} M_{P A}^{2} F_{P 0}^{2}=-\operatorname{tr}\left\{T^{A},\left\{\mathcal{M}, T^{B}\right\}\right\}<0\left|\frac{1}{3} u^{0}\right| 0>.
$$

The matrix $\mathcal{M}$ is decomposed in the following way along the matrices $T: \mathcal{M}=\left(m_{u}-m_{d}\right) T^{3}-$ $\frac{1}{\sqrt{3}}\left(2 m_{s}-m_{u}-m_{d}\right) T^{8}+\frac{1}{3}\left(m_{u}+m_{d}+m_{s}\right) \mathbf{1}$; the relation $\operatorname{tr}\left(T^{A} T^{B}\right)=\frac{1}{2} \delta_{A B}$ fixes the normalization of the matrices $T$. Defining

$$
B=-\frac{1}{3 F_{P 0}^{2}}<0\left|u^{0}\right| 0>, \quad \hat{m}=\frac{1}{2}\left(m_{u}+m_{d}\right),
$$


and neglecting electromagnetism and $\pi^{0}-\eta-\eta^{\prime}$ mixings, Eq. (63) decomposes into the following equations:

$$
M_{\pi^{+}}^{2}=M_{\pi^{0}}^{2}=2 \hat{m} B, \quad M_{K^{+}}^{2}=\left(m_{s}+m_{u}\right) B, \quad M_{K^{0}}^{2}=\left(m_{s}+m_{d}\right) B, \quad M_{\eta}^{2}=\frac{2}{3}\left(2 m_{s}+\hat{m}\right) B .
$$

One also verifies here the $S U(3)_{V}$ Gell-Mann-Okubo formula (in the isospin limit):

$$
4 M_{K}^{2}-3 M_{\eta}^{2}-M_{\pi}^{2}=0
$$

The masses $M_{P}$ and decay couplings $F_{P}$ are physical quantities; threfore, they do not depend on renormalization mass scales. However, the quark masses and the scalar densities are renormalized under interaction and depend on the renormalization mass scale, although their product does not. One must specify, when providing values for the quark masses, at which scale they have been evaluated. (Usually, they are chosen at a mass scale $\mu=2 \mathrm{GeV}$.) Also the ratios of quark masses are renormalization group invariant.

Numerically, one finds from the above formulas

$$
\frac{m_{s}}{\hat{m}}=26.0, \quad \frac{m_{u}}{m_{d}}=0.65, \quad \frac{m_{s}}{m_{d}}=21.5 .
$$

One deduces that $m_{d}>m_{u}$ and $m_{s} \gg \hat{m}$ (cf. also Eq. (15)). More precise results are obtained by incorporating electromagnetic effects $[5,30]$.

We consider now the vacuum expectation value of the scalar density $\left.u^{0}:<0\left|u^{0}\right| 0\right\rangle$. With respect to flavor $S U(3), u^{0}$ is a singlet operator. However, with respect to the chiral group $S U(3)_{L} \otimes S U(3)_{R}$, it has a more complicated structure. To display it, we introduce left-handed and right-handed quark and antiquark fields:

$$
\psi_{L}^{a}=\frac{1}{2}\left(1-\gamma_{5}\right) \psi^{a}, \quad \psi_{R}^{a}=\frac{1}{2}\left(1+\gamma_{5}\right) \psi^{a}, \quad \bar{\psi}_{L a}=\frac{1}{2} \bar{\psi}_{a}\left(1+\gamma_{5}\right), \quad \bar{\psi}_{R a}=\frac{1}{2} \bar{\psi}_{a}\left(1-\gamma_{5}\right) .
$$

In terms of them, $u^{0}$ is expressed as

$$
u^{0}=\bar{\psi}_{a} \psi^{a}=\bar{\psi}_{R a} \psi_{L}^{a}+\bar{\psi}_{L a} \psi_{R}^{a} .
$$

The left-handed and right-handed fields belong to representations of different groups, $S U(3)_{L}$ and $S U(3)_{R}$, respectively. One finds that $u^{0}$ belongs to the $\left(\overline{3}_{L}, 3_{R}\right)+\left(3_{L}, \overline{3}_{R}\right)$ representation of the chiral group. This is not the singlet representation. If the vacuum were invariant under chiral transformations, then $<0\left|u^{0}\right| 0>$ would be zero, according to the Wigner-Eckart theorem. Its nonvanishing is a sign that the vacuum is not invariant under chiral transformations and therefore chiral symmetry is spontaneously broken. In this case, $<0\left|u^{0}\right| 0>$ represents an order parameter of spontaneous chiral symmetry breaking. One has here an analogy with ferromagnetism, where the magnetization of the atoms has a nonzero value, resulting from the alignment of their spins along a particular direction, thus breaking the symmetry of the ground state, which corresponds to the disordered situation, where the spins are aligned randomly.

\section{Chiral perturbation theory}

After the successes of the predictions of low energy theorems, obtained in the chiral limit or in leading order of explicit chiral symmetry breaking, one naturally is interested by the calculation of corrective 
terms to the leading-order quantities. Essentially, there are two types of correction that arise: 1) quark mass term contributions; 2) many-Goldstone boson state contributions. The latter do not contribute at leading order because of damping factors coming from phase space coefficients. At nonleading orders, they are no longer negligible. They produce unitarity cuts with corresponding logarithmic functions of the momenta and the masses. Early calculations have been done by Li and Pagels [31, 32].

Calculation of the nonleading chiral symmetry corrections through a systematic perturbative approach has been proposed by Glashow and Weinberg [33] and by Dashen [34]. Initial calculations have been done with the use of the Ward-Takahashi identities [35]. But at higher-orders, this method becomes rapidly very complicated.

In this context, Weinberg has made several observations [36, 37]. 1) In spite of the fact that we are in the domain of strong interactions, the couplings of the Goldstone bosons with other particles and with themselves have turned out to be relatively weak in the final results of low-energy theorems. 2) These couplings are reminiscent of derivative coupling types. 3) All results of low-energy theorems could have been obtained (often more easily) by using phenomenological chiral invariant Lagrangians involving only the hadrons entering into the concerned processes; it would be sufficient to do the calculations at the tree level. Therefore, nonleading contributions to the low-energy theorems could be evaluated by calculating loop diagrams with the same Lagrangians.

How to construct chiral invariant hadronic Lagrangians? For this, one should know the chiral transformation properties of the hadronic fields and in particular of the Goldstone boson fields.

The problem of the realization of chiral symmetry through hadronic Lagrangians has been systematically studied first by Weinberg [36, 37]. Considering the case of $S U(2)_{L} \otimes S U(2)_{R}$ symmetry and denoting $\pi^{A}(A=1,2,3)$ the pion fields, which belong to the triplet representation of the flavor group (the isospin), one has

$$
\left[Q^{A}, \pi^{B}\right]=i \epsilon_{A B C} \pi^{C},
$$

while the transformation rule under the axial charges is left in a general form:

$$
\left[Q_{5}^{A}, \pi^{B}\right]=-i\left(\delta_{A B} f\left(\pi^{2}\right)+\pi^{A} \pi^{B} g\left(\pi^{2}\right)\right) .
$$

$\left(\epsilon_{A B C}=f_{A B C}\right.$ for $A, B, C=1,2,3$.) Conditions are obtained on the functions $f$ and $g$ from the action of the chiral algebra [Eqs. (22)] on the above equations and the use of the Jacobi identities. Equations are obtained for $f$ and $g$ that can be solved. The solution is not unique, due to the fact that any pseudoscalar field, having the same quantum numbers as the pion, can be considered as representing the pion field. These various definitions are related to each other by field transformations, which should not have any incidence on physical quantities. The general result is that the functions $f$ and/or $g$ are nonlinear functions of the pion fields. The chiral transformation rules of the pion fields are therefore nonlinear.

Similarly, one obtains the chiral transformation properties of massive hadronic states. The latter transform linearly, but the transformation coefficients are nonlinear functions of the pion fields.

To construct chiral invariant Lagrangians, one needs the introduction of chiral covariant derivatives, in which the role of connections is played by pion field dependent terms proportional to the derivatives of the pion fields. One thus finds deductively the property that the couplings of the Goldstone boson fields are of the derivative type.

Another approach to the construction of chiral invariant Lagrangians has been developed by Callan, Coleman, Wess and Zumino [38, 39], which also is more easily applicable to the $S U(3)_{L} \otimes$ $S U(3)_{R}$ symmetry case. Instead of considering the detailed form of the transformation of the Goldstone fields with respect to the action of the generators of the group, one here directly considers the 
transformation rules at the group element level. For this, one defines the composite field

$$
U(x)=e^{2 i \phi^{A}(x) T^{A} / F_{P 0}}
$$

where $\phi^{A}$ represent the Goldstone boson fields $(A=1, \ldots, 8), T^{A}$ the matrix representatives of the flavor group generators in the "fundamental representation and $F_{P 0}$ the Goldstone boson decay constants in the chiral limit. This object is recognized as an element of the $S U(3)_{L} \otimes S U(3)_{R}$ group, obtained from the application of the group operators on the identity element with parameters identified, up to multiplicative factors, with the Goldstone boson fields. The ordinary elements of the group are also of similar type, with parameters being now c-numbers. One has the transformation law

$$
U(x) \longrightarrow \Omega_{R}(x) U(x) \Omega_{L}^{+}(x),
$$

where $\Omega_{R}$ and $\Omega_{L}$ are elements of the groups $S U(3)_{R}$ and $S U(3)_{L}$, respectively. (A detailed derivation of Eq. (73) can be found in Refs. [6, 11].) They are considered as $x$-dependent to allow for the introduction of covariant derivatives. The latter are defined from Eq. (73) with external currents or sources playing the role of connections. The chiral invariant Lagrangian is constructed with the aid of the covariant derivatives, also including couplings to external scalar and pseudoscalar densities. This method of approach leads naturally, by means of the external sources, to the definitions of Green's functions of currents and densities. It has been adopted by Gasser and Leutwyler in their formulation of the chiral effective field theory of Goldstone bosons in the framework of QCD [40, 41].

Explicit chiral symmetry breaking is further introduced through mass terms of Goldstone bosons $[36,37]$ or through external sources representing quark masses [40, 41].

Next, one calculates loop corrections. Since Goldstone boson couplings are of the derivative type, each loop introduces, through its vertices, additional powers of the Goldstone boson momenta. At low energies, the latter are small, of the same order of magnitude as the Goldstone boson masses. Increasing the number of loops increases in turn the powers of the momenta and hence decreases the magnitude of the terms in comparison with the leading ones [42].

Therefore, one is led to a systematic power counting rule. The Goldstone boson masses squared $M_{P}^{2}$ (or equivalently the quark masses $\mathcal{M}$ ) and momenta squared, $q^{2}, k^{2}$, etc., are considered as perturbation theory parameters. Their higher powers will correspond to higher-order terms. Thus, the first-order corrections will involve at most one-loop diagrams. Chiral perturbation theory involves therefore expansions in quark masses and Goldstone boson momenta, rather than in coupling constants.

A crucial point that remains to be dealt with is renormalization. In order for the phenomenological hadronic Lagrangian to describe correctly the underlying theory (QCD), it is necessary to consider the most general chiral invariant Lagrangian together with all possible mass terms. Therefore, the latter will involve an infinite series of terms with an increasing number of derivatives and powers of the masses. It will involve, from the start an infinite number of unknown constants. However, the Lagrangian will be ordered according to the number of derivatives or powers of masses it involves. For instance, for the purely Goldstone boson Lagrangian one has the expansion [40, 41]

$$
\mathcal{L}=\mathcal{L}_{2}+\mathcal{L}_{4}+\cdots+\mathcal{L}_{2 n}+\cdots
$$

where the generic subscript $2 n$ designates the total number of the derivatives and of the power of the Goldstone boson masses contained in the related term. At each finite order of the expansion, there corresponds a finite number of independent terms. The leading-order contributions come from $\mathcal{L}_{2}$, those of the next-to-leading order from $\mathcal{L}_{2}+\mathcal{L}_{4}$, etc. 
Calculation of loops introduces divergences. These are then absorbed by the renormalization of the coupling constants of the higher-order parts of the Lagrangian. These coupling constants are called low energy constants (LEC). They also contain information about the contributions of the high-mass particles which do not appear explicitly in the Lagrangian. Thus, in the Lagrangian describing $\pi-\pi$ interaction, the $\rho$-meson is absent, but since the Lagrangian is describing only low-energy regions, the $\rho$-meson field is in some sense integrated out from the total Lagrangian of the theory and its effects are enclosed in the resulting LECs and the accompanying expressions.

At each finite order of the perturbation theory, there is only a finite number of LECs, which should be determined from experiment. Then the theory becomes predictive at that order.

The phenomenological chiral hadronic Lagrangians that are constructed from the previous principles define the chiral effective field theories designed to describe definite sectors of hadrons. Applications have concerned processes involving $\pi, K, \eta$ mesons [43-47], leading to improved precision with respect to the low-energy theorems. The method has been generalized to include baryon-Goldstone boson interactions [48-56].

In conclusion, the chiral effective field theory approach enables one to convert, at low energies and in the hadronic world, the highly complicated nonperturbative structure of QCD into a more transparent and familiar framework, with the sole aid of the symmetry properties of the theory.

\section{Anomalies}

Invariance properties of Lagrangians established at the classical level may sometimes not survive quantization. In QFT, the renormalization process deals with divergent quantities, and some conventional operations, like interchange of limits or translation of integration variables, may not be legitimate.

That question has been acutely raised with the study of the decay process of the neutral pion into two photons $\left(\pi^{0} \rightarrow \gamma \gamma\right)$. Using the usual Ward-Takahashi identities (WTI) of chiral symmetry, one derives a low-energy theorem stating that the corresponding decay width should vanish in the chiral limit, while, on experimental grounds, it is far from vanishing or being small. This paradox has been analyzed by Adler and Bell and Jackiw [57, 58], who have shown that in QED with massless fermions, coupled to external axial-vector currents and pseudoscalar densities, triangle diagrams, corresponding to fermion loops with two vector vertices and one axial-vector vertex, violate, through the WTI, the conservation of the axial-vector current. This result is unchanged, and more precisely unrenormalized, by higher-order radiative corrections [59]. Adler has proposed to include the above anomalous contribution in the divergence of the axial-vector current, which now reads, for massive fermions, [57]

$$
\partial^{\mu} j_{5 \mu}=2 i m \bar{\psi} \gamma_{5} \psi+\frac{e^{2}}{16 \pi^{2}} \epsilon^{\mu \nu \lambda \sigma} F_{\mu \nu} F_{\lambda \sigma}
$$

where $F$ is the electromagnetic field strength and $\epsilon$ the totally antisymmetric tensor $\left(\epsilon^{0123}=-\epsilon_{0123}=\right.$ $-1)$. Thus, in the presence of electromagnetism, the axial symmetry is explicitly broken, irrespective of the fermion masses, a result not evident at the classical level.

From a more general standpoint, the appearance of anomalies can be understood as coming from the necessity of renormalizing the fermionic determinant in the path integral formalism. There are no regularization schemes that preserve chiral invariance [60].

The general structure of anomalies in the case of non-Abelian currents has been studied by Bardeen and Wess and Zumino [61, 62]. The anomalous contributions appear through the presence of an odd number of axial-vector currents at the vertices of fermion one-loop diagrams, the number of 
vertices varying from three to five. Considering the case of one axial-vector current in the presence of other vector currents, the expression of its divergence becomes

$$
\begin{aligned}
\partial^{\mu} j_{5 \mu}^{A}=i \bar{\psi}\left\{\mathcal{M}, T^{A}\right\} \gamma_{5} \psi+\frac{N_{c}}{16 \pi^{2}} \epsilon^{\mu \nu \lambda \sigma} \operatorname{tr}_{\mathrm{f}}\left(T^{A} T^{B} T^{C}\right) F_{\mu \nu}^{B} F_{\lambda \sigma}^{C} \\
+\frac{g^{2} N_{f}}{32 \pi^{2}} \epsilon^{\mu \nu \lambda \sigma} \delta_{A 0} \sum_{a=1}^{N_{c}^{2}-1} G_{\mu \nu}^{a} G_{\lambda \sigma}^{a}, \quad A=0,1, \ldots, N_{f}^{2}-1,
\end{aligned}
$$

where the $F$ s are non-Abelian color singlet field strengths of external vector currents, playing the role of sources, the $G$ s the gluon field strengths, $N_{f}$ and $N_{c}$ the numbers of quark flavors and colors, respectively, $\operatorname{tr}_{\mathrm{f}}$ the trace in flavor space, and we have also included in the formula the contribtion of flavor singlet currents, with the convention $T^{0}=\mathbf{1}$.

We note that in the flavor singlet case, the anomaly receives also a contribution from the gluon fields. The latter is a dimension-four operator and, contrary to the usual mass terms, its effect is hard and could not be treated in general as a perturbation (cf. the comments after Eq. (11)). The presence of the anomaly in the divergence of the singlet axial-vector current explains the large mass value of the $\eta^{\prime}$ meson, which cannot be considered, in the chiral limit, as a Goldstone boson [63].

The presence of anomalies destroys the renormalizability and unitarity properties of field theories. This is not the case of QCD itself, since it does not contain axial-vector couplings; the anomalies may appear here only when external currents are considered. However, the situation is different with the Standard Model, which contains axial-vector couplings through its weak interaction part. A general theorem states that the anomalies will intrinsically disappear if the sum of the charges of all fermions of the theory is equal to zero. This is indeed the case of the Standard Model, where the sum of the charges of the quarks and of the leptons cancel to zero, thus confirming the consistency of the theory $[2,3,64]$.

Apart from their implication in filling a hole in the chiral Ward-Takahashi identities, the anomalies play also an important role in the analysis of the phases of a theory. Such an attempt has been undertaken by 't Hooft with the concept of 'naturalness' [65]. If physics is described by different effective theories at different scales, the anomaly structure should be preserved from one scale to the other when the transition concerns only one part of the particles. (This is a consequence of the zeroanomaly condition for the global theory.) This requirement can be applied in particular to QCD. The latter is described at high energies by elementary quark and gluon fields, while at low energies it is described by hadrons, leptons remaining the same. As a consequence of the global zero-anomaly condition, anomalies resulting from both descriptions should match each other. This is not a trivial requirement, since quarks belong to the triplet representation of the flavor group (in the case of $S U(3)_{V}$ ), while hadrons belong to higher representations, like octets, decuplets, etc. The net result of the investigation shows that when the number of quark flavors is greater than two, only the phase of spontaneous chiral symmetry breakdown can fulfill the anomaly matching condition [65-68]. The above analysis brings therefore an indirect theoretical proof of spontaneous chiral symmetry breaking in QCD with more than two quark flavors. This result has been completed with the proof of Vafa and Witten that in vector-like gauge theories, such as QCD, the vector flavor group and parity cannot be spontaneously broken $[69,70]$.

Acknowledgments. I thank the Organizing Committee of the Conference Quark Confinement and the Hadron Spectrum XII for inviting me to give this lecture. I also thank Véronique Bernard and Bachir Moussallam for discussions and useful information. 


\section{References}

[1] S. Coleman, Aspects of symmetry (Cambridge University Press, Cambridge, UK, 1985).

[2] C. Itzykson and J.-B. Zuber, Quantum field theory (McGraw-Hill, New York, 1980).

[3] M. Kaku, Quantum field theory: A modern introduction (Oxford University Press, New York, USA, 1993).

[4] M. E. Peskin, Les Houches Lectures, 1982, SLAC-PUB-3021.

[5] J. Gasser and H. Leutwyler, Phys. Rep. 87, 77 (1982).

[6] A. V. Manohar, Lect. Notes Phys. 479, 311 (1997).

[7] S. Scherer, Adv. Nucl. Phys. 27, 277 (2003).

[8] J. Gasser, Lect. Notes Phys. 629, 1 (2004).

[9] G. Ecker, arXiv: hep-ph/0604165.

[10] V. Bernard and U.-G. Meissner, Ann. Rev. Nucl. Part. Phys. 57, 33 (2007).

[11] B. Kubis, arXiv: hep-ph/0703274.

[12] N. Brambilla et al., Eur. Phys. J. C 74:2981, 1 (2014).

[13] G. Preparata and W. I. Weisberger, Phys. Rev. 175, 1965 (1968).

[14] J. Goldstone, Nuovo Cimento 19, 154 (1961).

[15] J. Goldstone, A. Salam and S. Weinberg, Phys. Rev. 127, 965 (1962).

[16] Y. Nambu, Phys. Rev. Lett. 4, 380 (1960).

[17] Y. Nambu and G. Jona-Lasinio, Phys. Rev. 122, 345 (1961).

[18] M. Gell-Mann and M. Lévy, Nuovo Cimento 16, 705 (1960).

[19] K. A. Olive et al., Particle Data Group, Chin. Phys. C 38, 090001 (2014).

[20] M. L. Goldberger and S. B. Treiman, Phys. Rev. 111, 354 (1958).

[21] V. Baru et al., Phys. Lett. B 694, 473 (2011).

[22] J. C. Ward, Phys. Rev. 78, 182 (1950).

[23] Y. Takahashi, Nuovo Cimento 6, 371 (1957).

[24] C. G. Callan and S. B. Treiman, Phys. Rev. Lett. 16, 153 (1966).

[25] S. Weinberg, Phys. Rev. Lett. 17, 616 (1966).

[26] Y. Tomozawa, Nuovo Cimento A 46, 707 (1966).

[27] W. I. Weisberger, Phys. Rev. Lett. 14, 1047 (1965).

[28] S. L. Adler, Phys. Rev. Lett. 14, 1051 (1965).

[29] M. Gell-Mann, R. J. Oakes and B. Renner, Phys. Rev. 175, 2195 (1968).

[30] H. Leutwyler, Phys. Lett. B 378, 313 (1996).

[31] L.-F. Li and H. Pagels, Phys. Rev. Lett. 26, 1204 (1971).

[32] H. Pagels, Phys. Rep. 16, 219 (1975).

[33] S. L. Glashow and S. Weinberg, Phys. Rev. Lett. 20, 224 (1968).

[34] R. F. Dashen, Phys. Rev. 183, 1245 (1969).

[35] R. F. Dashen and M. Weinstein, Phys. Rev. Lett. 22, 1337 (1969).

[36] S. Weinberg, Phys. Rev. 166, 1568 (1968).

[37] S. Weinberg, Phys. Rev. 177,2604 (1969).

[38] S. R. Coleman, J. Wess and B. Zumino, Phys. Rev. 177, 2239 (1969).

[39] C. G. Callan, Jr., S. R. Coleman, J. Wess and B. Zumino, Phys. Rev. 177, 2247 (1969).

[40] J. Gasser and H. Leutwyler, Ann. Phys. (N.Y.) 158, 142 (1984).

[41] J. Gasser and H. Leutwyler, Nucl. Phys. B 250, 465 (1985).

[42] S. Weinberg, Physica A96. 327 (1979). 
[43] J. Gasser and H. Leutwyler, Nucl. Phys. B 250, 517 (1985).

[44] J. Gasser and H. Leutwyler, Nucl. Phys. B 250, 539 (1985).

[45] V. Bernard, N. Kaiser and U.-G. Meissner, Nucl. Phys. B 357, 129 (1991).

[46] G. Colangelo, J. Gasser and H. Leutwyler, Nucl. Phys. B 603, 125 (2001).

[47] J. Bijnens, Prog. Part. Nucl. Phys. 58, 521 (2007).

[48] J. Gasser, M. E. Sainio and A. Švarc, Nucl. Phys. B 307, 779 (1988).

[49] E. E. Jenkins and A. V. Manohar, Phys. Lett. B 255, 558 (1991).

[50] G. Burdman and J. F. Donoghue, Phys. Lett. B 280, 287 (1992).

[51] M. B. Wise, Phys. Rev. D 45, R2188 (1992).

[52] T.-M. Yan et al., Phys. Rev. D 46, 1148 (1992).

[53] V. Bernard, N. Kaiser, J. Kambor and U.-G. Meissner, Nucl. Phys. B 388, 315 (1992).

[54] P. L. Cho, Nucl. Phys. B 396, 183 (1993).

[55] U.-G. Meissner, arXiv: hep-ph/9711365.

[56] T. Becher and H. Leutwyler, Eur. Phys. J. C 9, 643 (1999).

[57] S. L. Adler, Phys. Rev. 177, 2426 (1969).

[58] J. S. Bell and R. Jackiw, Nuovo Cimento A60, 47 (1969).

[59] S. L. Adler and W. A. Bardeen, Phys. Rev. 182, 1517 (1969).

[60] K. Fujikawa, Phys. Rev. Lett. 42, 1195 (1979).

[61] W. A. Bardeen, Phys. Rev. 184, 1848 (1969).

[62] J. Wess and B. Zumino, Phys. Lett. B 37, 95 (1971).

[63] G. 't Hooft, Phys. Rep. 146, 357 (1986).

[64] C. Bouchiat, J. Iliopoulos and P. Meyer, Phys. Lett. B 38, 519 (1972).

[65] G. 't Hooft, NATO Sci. Ser. B 59, 135 (1980).

[66] Y. Frishman, A. Schwimmer, T. Banks and S. Yankielowicz, Nucl. Phys. B 177, 157 (1981).

[67] S. R. Coleman and B. Grossman, Nucl. Phys. B 203, 205 (1982).

[68] S. R. Coleman and E. Witten, Phys. Rev. Lett. 45, 100 (1980).

[69] C. Vafa and E. Witten, Nucl. Phys. B 234, 173 (1984).

[70] C. Vafa and E. Witten, Phys. Rev. Lett. 53, 535 (1984). 